\title{
Non-convulsive status epilepticus resistant to benzodiazepines
}

\author{
J H LIVINGSTON AND J K BROWN
}

Royal Hospital for Sick Children, Edinburgh

SUMMARY We describe the failure of an intravenous benzodiazepine to control non-convulsive status epilepticus occurring in six patients with the Lennox-Gastaut syndrome. In one patient the benzodiazepine induced a paradoxical response with clinical and electroencephalographic seizures.

Non-convulsive status epilepticus is a rare but serious complication of seizure disorders in childhood, particularly in the age dependent epileptic encephalopathies, such as the Lennox-Gastaut syndrome. ${ }^{1}$ The effects of non-convulsive status on cerebral metabolism are uncertain. In children with intractable seizure disorders, however, the incidence and degree of subsequent mental retardation is greater in those who have had one or more episode of non-convulsive status compared with those who have had no episodes. ${ }^{12}$ In addition, during these episodes, the children are very slow to react to any stimuli, are unable to concentrate, have no ability to learn, become disorientated and confused, and often lose speech. Episodes of nonconvulsive status may last from a few hours up to several months. ${ }^{3}$ For these reasons such episodes are regarded by many as a paediatric emergency requiring aggressive treatment.

While it is recognised that non-convulsive status may be drug resistant, in general intravenous benzodiazepines have been considered to be the drugs of choice for this complication..$^{2-4}$ Gastaut has gone as far as to say that all cases of absence status respond within a few minutes to intravenous benzodiazepines. ${ }^{5}$

We report here our experience with seven patients presenting in non-convulsive status treated with benzodiazepines.

\section{Patients and methods}

The patients reported here formed part of a group of 40 patients with intractable seizure disorders in whom testing of benzodiazepine sensitivity has been carried out. Nine out of the forty $(23 \%)$ had an electroclinical diagnosis of the Lennox-Gastaut syn- drome, and seven of these nine presented with at least one episode of non-convulsive status. These seven patients had testing of benzodiazepine sensitivity performed during the acute episodes of status and form the subject of this paper.

Details of the seven patients are shown in the Table. All patients were attending the paediatric epilepsy service at the Royal Hospital for Sick Children, Edinburgh. There were four boys and three girls. The mean age of presentation was 7 years (range 3-13 years). The aetiology in all cases was unknown, but in one patient it was presumed to be prenatal in origin as he showed dysmorphic features with microcephaly. The usual seizure type in most patients comprised drop attacks, complex absences, myoclonic jerks, and tonic seizures. All patients had presented with increased frequency in their usual seizures, followed by onset of unresponsiveness, drooling, and prolonged absences, in some cases associated with loss of speech and ataxia.

In all the patients the electroencephalogram (EEG) showed continuous or almost continuous slow spike or polyspike and wave of varying degrees of symmetry and synchrony. The duration of status as determined by history ranged from two days to five weeks (mean 10 days).

All patients were given intravenous benzodiazepines during continuous EEG monitoring. Five patients were given diazepam in a dose of $0 \cdot 2-0 \cdot 3$ $\mathrm{mg} / \mathrm{kg}$, one was given clonazepam in a dose of 0.02 $\mathrm{mg} / \mathrm{kg}$, and one was given both clonazepam and diazepam.

\section{Results}

Examples of the EEG response to intravenous benzodiazepines are shown in the Figure. Only one 


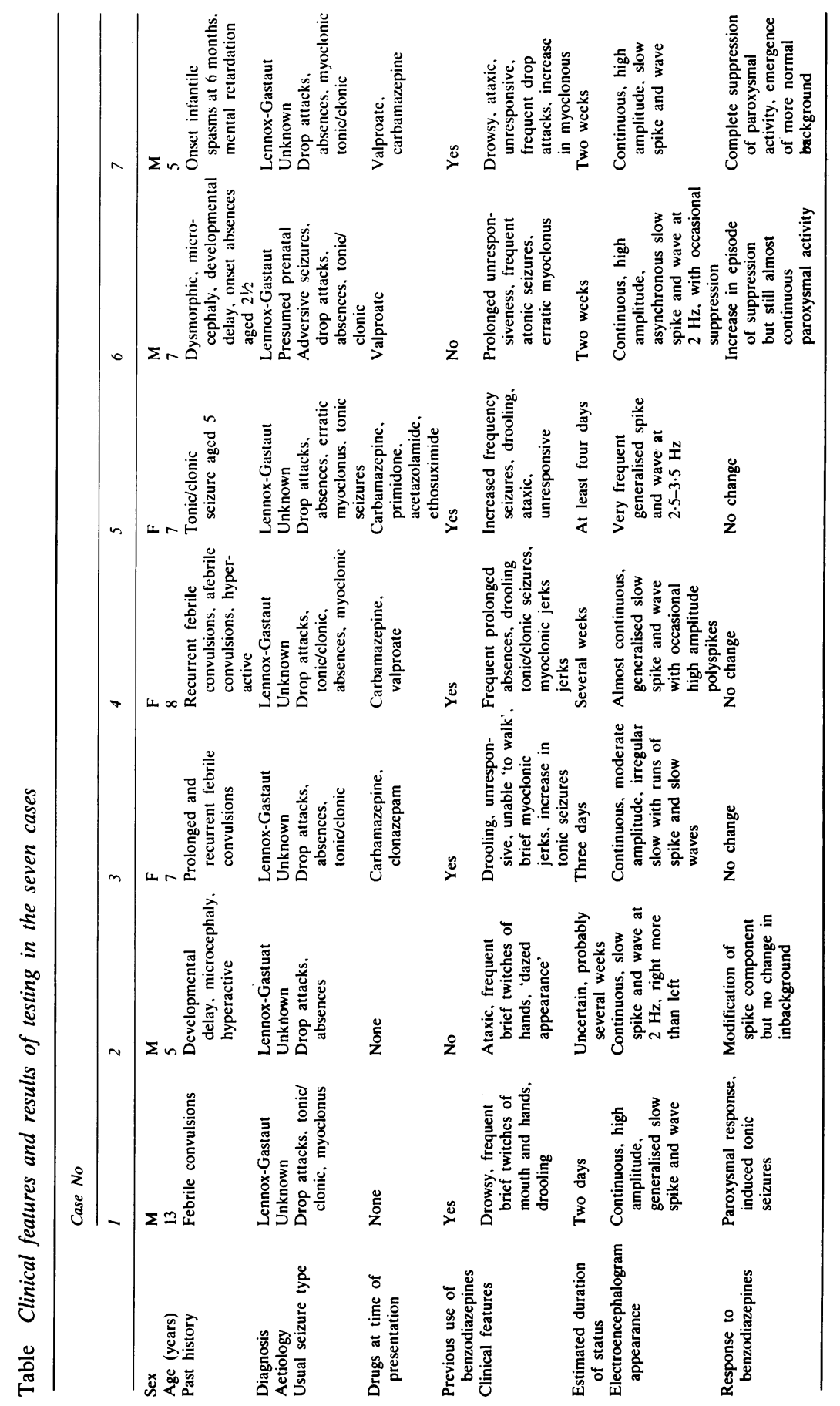

을

蛋

들

$\frac{0}{\overline{0}}$

@

$\vec{\circ}$

$\overrightarrow{\vec{\omega}}$

응

i

$\stackrel{?}{\prime \prime}$

운 
(a) Before diazepam

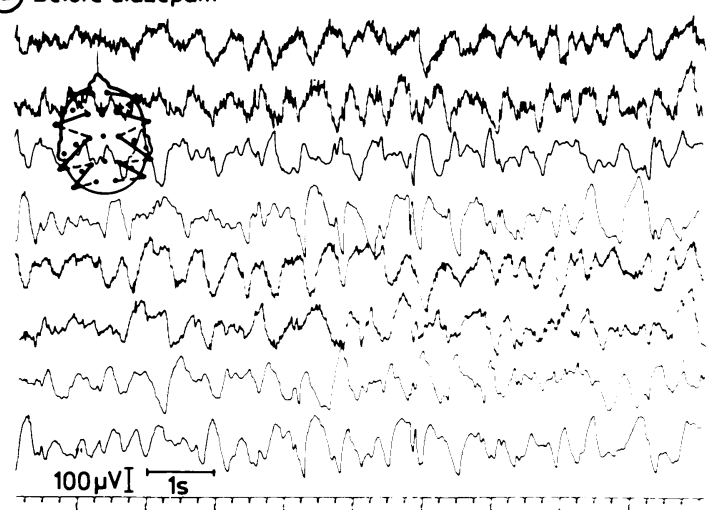

After diazepam

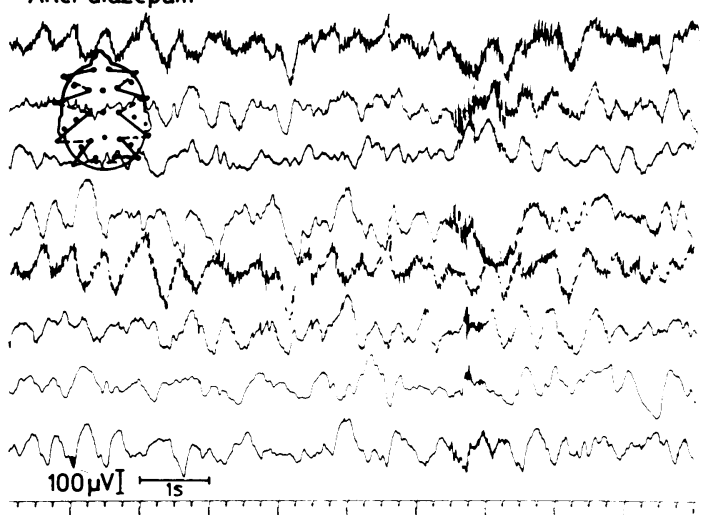

(b)
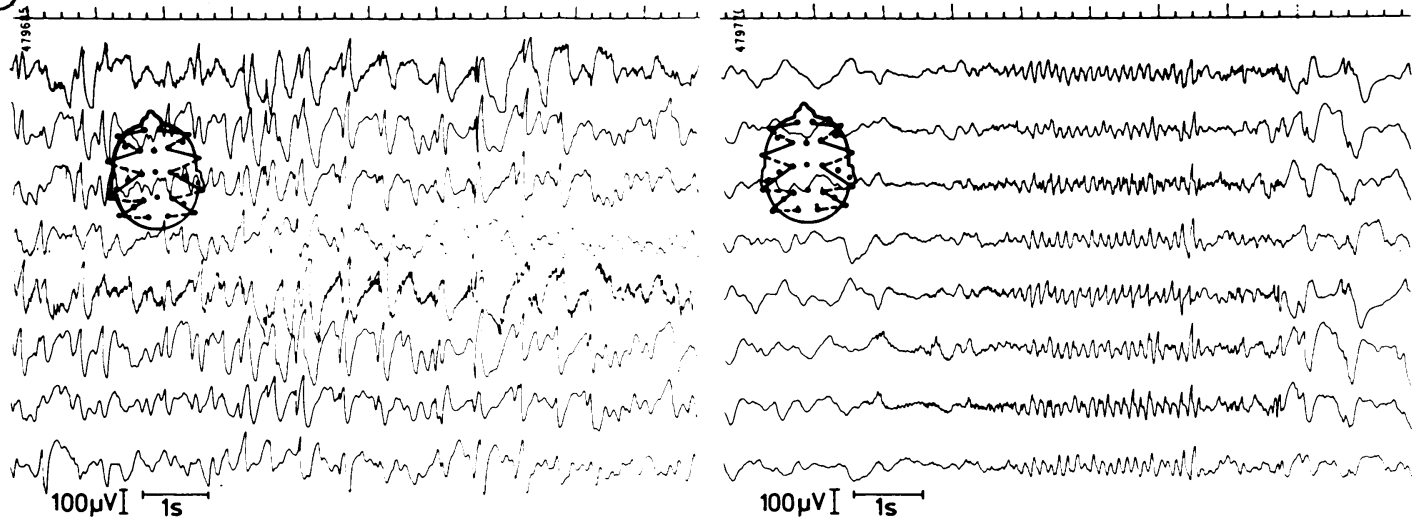

Figure (a) Example of non-response to administration of diazepam in case 3; (b) Paradoxical response occurring in case 1 associated with tonic seizure.

patient showed a positive response with complete abolition of paroxysms and improvement in clinical state after treatment with benzodiazepines. One patient (case 6) showed a change in his EEG with an increase in the number of episodes of suppression but still with virtually continuous paroxysmal spike and wave activity and no associated clinical improvement. One patient showed some modification of the spike component but continued with high amplitude slow wave background activity and no clinical change. One patient (case 1) had a paradoxical response with the onset 10 minutes after administration of benzodiazepines of paroxysms of rhythmic spikes at 10 per second followed by spike and wave. On each occasion these paroxysms were associated with a tonic seizure. Three patients showed no change in their EEG or clinical state.

\section{Discussion}

These results suggest that the benzodiazepines may not be the most effective treatment for nonconvulsive status. It is well known, however, that the effects of the benzodiazepines are unpredictable and that patients' sensitivity to the drugs may change with time, depending on such variables as current anticonvulsive treatment and seizure control.

Lagenstein reported a $57 \%$ response rate to intravenous benzodiazepines in 56 patients with a diagnosis of petit mal status. They also reported, however, a high relapse rate. ${ }^{6}$ In contrast, Tassinari found that only $15 \%$ of children with non-convulsive status associated with the Lennox-Gastaut syndrome showed EEG or clinical response to intravenous benzodiazepines. ${ }^{7}$

Benzodiazepines act by enhancing $\gamma$-aminobutyric acid (GABA) mediated inhibition in the central nervous system by their action on the GABAbenzodiazepine/chloride ionophore complex. There are at least two pharmacologically distinct benzo- 
diazepine receptors, and type 2 probably consists of several subunits. A recent hypothesis suggests that each benzodiazepine receptor has four different binding sites for the benzodiazepines and each of these sites can exist in three different conformations. It is thus theoretically possible for the benzodiazepines to increase, decrease, or have no effect on gabinergic transmission. ${ }^{8}$

In addition, other anticonvulsants are known to interact directly at the benzodiazepine receptor or to have other effects on GABA mediated inhibition. As well as the conformational and interactional changes that may occur in benzodiazepine receptors, it is also known that changes in receptor numbers may occur with chronic treatment, and thus both down regulation and up regulation of receptors have been described. ${ }^{9}$

Five of the seven patients in this group had previously been on oral treatment with benzodiazepines and thus these mechanisms may explain their subsequent lack of response. Two of the patients, however, had no previous exposure to them and clearly down regulation of receptors could not explain the lack of response in these patients. It does seem, however, that the chronicity of the seizure disorder, together with the need for many different anticonvulsants taken over long periods, seem to make negative or paradoxical responses more likely.

The benzodiazepines are the most effective treatment for convulsive status epilepticus available and from this small series we would not suggest that they should be avoided in the treatment of nonconvulsive status. These results illustrate, however, that many patients with non-convulsive status will not respond to them in the acute stage. We would emphasise the importance of continuous EEG monitoring during the treatment of these patients to assess the effects and watch for unusual or paradoxical responses that are more common in this group of patients. If diazepam has failed we would suggest a trial of clonazepam, although in our experience we have not seen anyone in nonconvulsive status who has responded to clonazepam that did not respond to diazepam. These states are often very drug resistant, and many different therapeutic manoeuvres have been tried. It has been suggested that infusion of chlormethiazole may enable benzodiazepine sensitivity to return, but this has not been our experience.
In patients who have become resistant to benzodiazepines after a previous response a drug holiday of several weeks may allow sensitivity to return. This approach would only have been applicable, however, to one of our patients in this study, as six of the seven were not on benzodiazepines at the time of testing. Clobazam has been suggested to be superior to the other benzodiazepines for chronic oral treatment. Our clinical impression is that, while it does seem more effective in other types of intractable seizure disorders, it has not proved to be an advance in patients with the Lennox-Gastaut syndrome.

If benzodiazepines have failed to control nonconvulsive status we would suggest immediate treatment with either regular paraldehyde, infusion of chlormethiazole, or possibly infusion of barbiturates or steroids.

Although there is increasing concern about the possible brain damage caused by recurrent bouts of non-convulsive status, treatment of these states remain frustratingly difficult with the current anticonvulsants at our disposal.

\section{References}

' Gastaut H, Roger J, Soulayorol R, et al. Childhood epileptic encephalopathy with diffuse slow spike-waves (otherwise known as "petit mal variant") or Lennox syndrome. Epilepsia 1966;7:139-79.

2 Doose H, Volzke E. Petit mal status in carly childhood and dementia. Neuropadiatrie 1979;10:10-4.

${ }^{3}$ Roger J, Lob H, Tassinari CA. Status epilepticus. In: Vinken PJ, Bruyn GW, eds. Handbook of clinical neurology. Amsterdam: North Holland Publishing Company, 1974:145-88.

${ }^{4}$ Congdon PJ, Forsythe WI. Intravenous clonazepam in the treatment of status epilepticus in children. Epilepsia 1980;21: 97-102.

${ }^{5}$ Gastaut H. Classification of status epilepticus. Adv Neurol 1983;34:15-36.

${ }^{6}$ Langenstein I, Iffland E. Intravenous therapy of petit mal status with diazepam and clonazepam. EEG EMG 1977;8:82-8.

7 Tassinari CA, Danicle O, Michelucci R, Bureau M, Dravet C, Roger J. Benzodiazepines: efficacy in status epilepticus. $A d v$ Neurol 1983;34:465-76.

${ }^{8}$ Sieghart W. Benzodiazepine receptors: multiple receptors or multiple conformations. J Neural Transm 1985;63:191-208.

${ }^{9}$ Sher PK. Reduced benzodiazepine receptor binding in cerebral cortical cultures chronically exposed to diazepam. Epilepsia 1983;24:313-20.

Correspondence to Dr J H Livingston, Department of Paediatric Neurology, Royal Hospital for Sick Children, Sciennes Road, Edinburgh EH9 1LF.

Received 15 July 1986 